\title{
Method for identification of perlite-class steel microstructure parameters using metallographic images
}

\author{
R.G. Magdeev ${ }^{1}$, A.G. Tashlinskiy ${ }^{1}$ \\ ${ }^{a}$ Ulyanovsk State Technical University, Severnii Venets, 32, 432027, Ulyanovsk, Russia
}

\begin{abstract}
A method for finding the microstructural parameters of low-carbon steel using its metallographic images is proposed. It allows to determine the following parameters: the ratio of perlite to ferrite phases, the parameters of grains of crystallites and their mutual arrangement; the degree of granularity of the pearlite phases. The method is aimed at predicting the strength characteristics of steel samples and consists of several stages. The preprocessing step involves color reduction, refinement of the area of interest, noise filtering, illumination refinement and histogram equalization. The segmentation of the image is associated with the search for the size, area and convex shell of grains. The stage of finding the microstructural parameters is based on the stochastic gradient-based estimation of the parameters of the segmented objects. Examples of analysis of steel oil pipeline samples with a forecast of their strength characteristics are given.
\end{abstract}

Keywords: metallographic image; digital image processing; stochastic gradient-based estimation; convex shell; steel microstructure parameters; grains of crystallites; perlite; ferrite

\section{Introduction}

Low-carbon (with a carbon content of less than 0.8\%), low-alloy (less than 5\% of alloying elements) steels, pearlite hypereutectoid steels are the main products of ferrous metallurgy. They are used for manufacturing a wide range of tools and parts with increased strength and elastic properties, pipelines, steel trusses, etc. [1,2].

One of the key problems in manufacturing and operation of steel products is the control of the compliance of these products with the required characteristics (strength, residual life, possibility of use under certain conditions, etc.), which is primarily ensured by the characteristics of the steel itself. The mechanical properties include hardness, strength, viscosity, elasticity, plasticity, etc. These properties are determined by chemical composition, macro- and microstructure, production and processing methods [3].

Steel microstructure is a combination of a large number of grains in the form of adjacent crystallites differing in size, shape, and spatial orientation. All microstructures of low-carbon and low-alloy steels contain a perlite-eutectoid mechanical mixture of ferrite and cementite [2]. Pre-eutectoid steel has a lamellar structure consisting of alternating plates of ferrite and cementite [4]. An essential feature of the microstructure is the presence of internal boundaries separating the grains in the metal.

Usually, images of microstructures are obtained by means of a digital metallographic microscope at various magnifications [5]. In the images one can see different phases, outlines of the grains and their mutual arrangement. The images used in this work were obtained from steel pipelines for transferring petroleum products using the LOMO BIOLAM M-1 laboratory research microscope with a special nozzle and installed MC-3 digital camera with resolution 1200x900 pixels. Fig.1 shows an example of an image of the microstructure of a metal of a 17GS pipeline at 200x magnification.

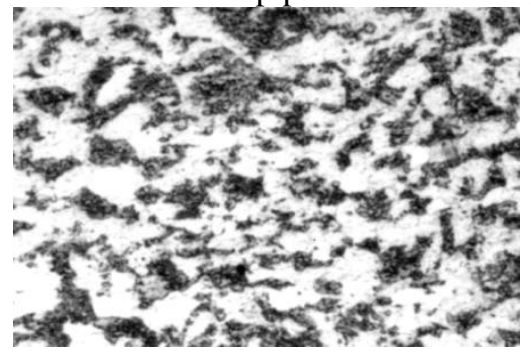

Fig. 1. Example of steel 17GS microstructure.

Metallographic methods for detecting and determining the grain size of steels and alloys are established by GOST 5639 [6]. These methods are: visual comparison of grains with scale templates, counting the number of grains per unit surface of the section, counting the intersections of grain boundaries by straight lines, measuring the length of the chords with the determination of the relative fraction of grains of a certain size, and the ultrasonic method based on the dependence of the attenuation of ultrasonic oscillations on grain sizes.

Numerous studies have shown the relationship between the parameters of the microstructure and the mechanical properties of metals and alloys [5, 7-10, etc.]. The dependence of the strength characteristics of cold-rolled steels at the production stage on the change in the microstructure was investigated in [11, 12, 13]. In practice, most microstructural studies are carried out visually by experts, which does not allow for an objective assessment of their reliability. Therefore, the development of a technique capable of automating the process of obtaining microstructural characteristics on the basis of digital image processing methods and algorithms seems to be an urgent task. Unfortunately, a small number of papers have been devoted to solutions of this problem, in particular $[14,15]$. However, they do not consider the evaluation of the properties of steels based on the 
microstructural characteristics found, which is in demand both at the production stage and after long-term operation. In addition to the basic microstructural characteristics, parameters such as the degree of ordering of orientations of the perlite grains and the degree of granularity of the pearlite phases are also important for determining the degree of metal fatigue.

\section{Basic microstructural parameters of steel}

Shape and arrangement of grains in low-carbon and low-alloy steels are subjected to certain regularities associated with the solidification of the metal and its transformation during processing and operation. In accordance with GOST 8233 [11], the basic parameter is the ratio of the pearlite and ferritic phases of the microstructure of metal. For a ferrite-perlite the ratio can very between 0 and $95 \%$. This range of ratios can be covered using templates by various image processing means.

One of the main geometric parameters of the microstructure of a metal is the grain size, which is its mean diameter. The size $d_{i}$ of the $i$-th grain is characterized by the arithmetic average of the longitudinal (maximum) size $W_{i}$ and the transverse (minimum) grain size $H_{i}$ The average size $\bar{d}$ is found as the average of all the changed grains.

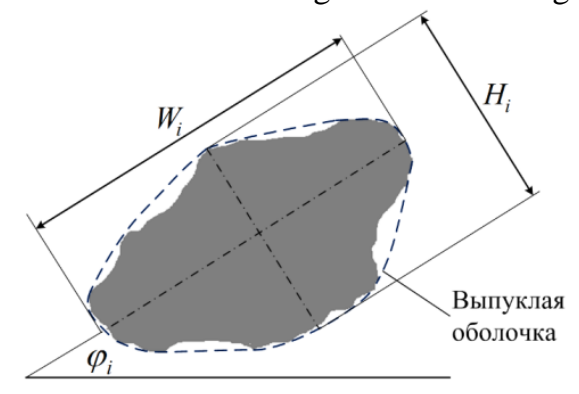

Fig. 2. Metal grain geometric parameters.

A correlation was found between the average grain size $\bar{d}$ and the yield and strength limits, which are described by the HallPetch dependence [2]: $\sigma_{S}=\sigma_{S_{0}}+g \sqrt{\bar{d}}$, where $\sigma_{S}$ - the yield strength; $\sigma_{S_{0}}$ - the yield strength of the initial (in the production of steel); $g$ - constant coefficient determined by the steel grade.

Large dispersion of grain sizes adversely affects the uniformity of mechanical and operational properties of products. To take into account this circumstance, the spread parameter of the grains is used [2]. It was shown in [5] that the grain size is a random variable that has a normal distribution law, accordingly the spread of grain sizes corresponds to the mean square deviation of the Gaussian distribution.

Over time, with sufficient external forces, the plastic deformation covers the entire volume of the polycrystalline. As a result, the grains get an elongated shape in the direction of the most intense flow of the metal. Simultaneously with the change in the shape of the grains during the deformation, the crystallographic axes of the individual grains rotate in the direction of the greatest deformation, which leads to anisotropy of the properties of the metal.

To control the microstructural characteristics at the stages of metal rolling, the Tretyakov method [12] based on empirical formulas for determining the mechanical characteristics of steels and alloys depending on the degree of deformation is used. In particular, for cold rolling, the conditional yield strength $\sigma_{\mathrm{CS}}$ is calculated by the formula: $\sigma_{\mathrm{CS}}=\sigma_{\mathrm{CS}_{0}}+A \varepsilon^{b}$, where $\sigma_{\mathrm{CS}_{0}}$ is the conditional yield strength in the initial state; $\varepsilon$ - degree of metal deformation, $\% ; A$ and $b$ are constant coefficients determined by the steel grade. However, the above relation does not take into account the anisotropic properties of microstructures, for which the average coefficients of anisotropy of the grain shape are: $\bar{k}_{\text {begin }}=\bar{W}_{\text {begin }} / \bar{H}_{\text {begin }}$ before and $\bar{k}_{\text {end }}=\bar{W}_{\text {end }} / \bar{H}_{\text {end }}=$ $\left(\bar{k}_{\text {begin }}+\varepsilon\right) /(1-\varepsilon)$ after deformation.

The degree of orderliness of orientations of the perlite grains in the investigated region of the steel microstructure is characterized by the directivity vector and the ordering coefficient. For a particular grain, the vector of orientation $\vec{K}_{i}^{\text {dir }}=d_{i}$. $\exp \left(-j \phi_{i}\right)$, i.e. the direction of the vector coincides with the direction of the longitudinal axis of the perlite grain (fig. 2). The general vector of the grain orientation is:, $\vec{K}_{\Sigma}^{\text {dir }}=\sum_{i=1}^{n} \vec{K}_{i}^{\text {dir }}$, and the ordering coefficient is: $k_{\text {ord }}=\left|\vec{K}_{\Sigma}^{\text {dir }}\right| /(n \bar{d})$, where $n-$ the number of grains is.

Other important characteristics of the metal microstructure affecting the mechanical properties of steel are the ratio of perlite to ferrite $k_{\mathrm{PF}}, \%$, and the degree of granularity of the pearlite phases $k_{\text {grit }}, \%$.

\section{Stages of metallographic image processing method}

The proposed technique for identifying microstructure parameters can be divided into three main stages.

Preliminary processing of images under study aimed at increasing the accuracy and reliability of finding the microstructural parameters of perlite grains. It consists of the following operations: color reduction of the image in order to simplify subsequent calculations area of interest extraction is aimed at excluding low-information areas of the image, filtering the image to compensate for high-frequency distortions caused by the peculiarities of the metallographic microscope path, brightness refinement compensating for uneven illumination of the microsection, and histogram equalization.

Segmentation on metallographic images of areas corresponding to perlite grains according to which their microstructural parameters are further located. It is achieved by the following procedures: segmentation, aimed at identifying areas of pearlite 
Image Processing, Geoinformation Technology and Information Security / R.G. Magdeev, A.G. Tashlinskiy

grains, mathematical morphology for eliminating internal discontinuities in grain images and excluding from the further analysis of small objects, isolating external boundaries and constructing convex shells of grains for the subsequent calculation of microstructural parameters.

Estimation of microstructural parameters of perlite grains, including the formation of adaptive templates for finding object parameters, Gaussian filtration of convex shells of isolated grains and formed templates for the purpose of expanding the working range of stochastic gradient descent procedures $[13,14]$ used to estimate the microstructural parameters of grains, finding particular and integral microstructural parameters of perlite grains and the degree of granularity of pearlite phases.

Below the implementation of the above steps of the methodology is briefly considered.

\subsection{Image proprocessing}

Image color reduction. In the formulated problem, the informative component of color is small, so vector-based (color) images are advisable to translate into levels of gray [15]. It should be noted that there are color models of images in which the luminance component is already separated into a separate stream: HSV, HSL, YUV, etc. The YUV model in the recommendation ITU-R BT.601, whose brightness component is calculated according to the formula [16]:

$$
z_{\text {grey }}(x, y)=0.299 z_{r}(x, y)+0.587 z_{g}(x, y)+0.114 z_{b}(x, y)
$$

where $z_{r}(x, y), z_{g}(x, y), z_{b}(x, y)$ - values of the red, green, and blue components of the pixel with the coordinates $(x, y)$; $z_{\text {grey }}(x, y)$ pixel brightness value obtained as a result of monochromization. This procedure is performed for all sample counts.

Area of interest extraction is aimed at excluding from the further processing of low-information areas, the presence of which is related to the specificity of the imaging by a metallographic microscope, which causes the image to be formed approximately in the form of a circle. The analysis $[9,17]$ showed that the brightness of the non-informative fragment differs significantly from the brightness of the informative fragment, and is usually $2.5-3 \%$ of the maximum brightness. With this in mind, the center and the radius of the circle are sought. Further processing of only the highly informative image area reduces the requirements for computational resources. In a particular implementation of the technique, after finding the image processing area for visual convenience, the brightness of the samples is inverted. Fig. 3, a shows examples of the selected processing area of two metallographic images.

Image filtering is aimed at elimination of brightness distortions caused by the imperfections of the optical detectors of the metallographic microscope. In images they appear as small (one - two pixels), but significant (up to 45\% to neighboring pixels) brightness increase. The nature of the appearance of distortions is due to optics and reflections during photography. To eliminate their influence on the final result, nonlinear median filtration was used [19]. The size of the median filter window for metallographic images is usually $3 \times 3$ or $5 \times 5$.

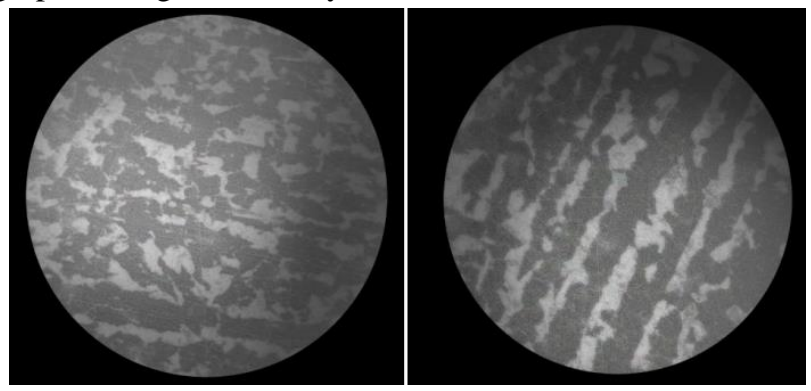

a)

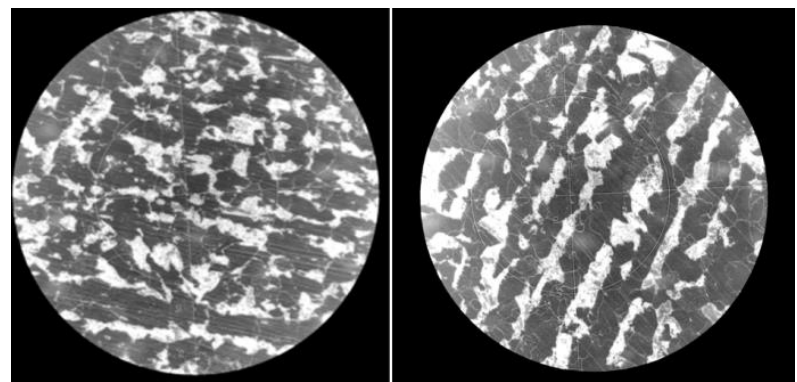

b)

Fig. 3. Examples of two metallographic images: a) after area of interest extraction, б) after image equalization.

Illumination refinement is used for lighting unevenness compensation. A typical example of distortion of this kind in metallographic images is a shadow. In this case, the image $Z$ can be represented as: $Z=X \cdot \gamma$, where $X$ - an undistorted image, $\gamma$ - illumination coefficient. One can obtain an approximate illumination map by applying a Gaussian filter with a large blur radius (about $5 \%$ of the highly informative image area). The restored image is looked for as: $\hat{X}=\exp (\log (Z)-\log (\hat{\gamma})), \operatorname{which}$ allows not only to align the image with the level of illumination, but also to perform gradient transformations [22].

Histogram equalization aligns the intensities with the aim of improving the quality of the display. To carry out the equalization, the conversion is performed: $z_{\mathrm{ekv}}(x, y)=f(z(x, y))$, where $z(x, y)-$ brightness value in the pixel with coordinates $(x, y)$ of the original image, $z_{\mathrm{ekv}}(x, y)$ - brightness value of the converted image, and $f(z)$ is the single-valued monotonically increasing conversion function.

Fig. $3 b$ shows examples of images after the preprocessing phase. 
At this stage, the problem of isolating individual perlitic spots is solved with a view to their further analysis. To solve this problem, a growing areas method is used [16] with preliminary procedures for binary segmentation and mathematical morphology of the resulting binary image.

The problem of segmentation of the perlite spots is solved using the image binarization procedure based on the histogram analysis of the image, taking into account the sizes of the desired regions.

Morphological closing (in particular, with 5x5 kernel) is aimed at eliminating objects less than the specified window and filling the gaps that are inside the images of pearlite spots.

Individual perlite grain segmentation is based on the method of growing areas [19] as follows: on a binary image is a pixel belonging to perlite. If the neighboring pixel of the image also belongs to the perlite - the decision is made whether this pixel belongs to this spot, and it is appropriately marked. The procedure continues until all adjacent pixels are labeled or belong to ferrite.

Perlite grain boundaries estimation. The boundaries of the selected objects are found via algorithms for the sequential construction of contours, which are characterized by high speed, absence of discontinuities and "extra" boundaries with low computational complexity. In particular, the recursive algorithm of the "beetle" was used [10, 20]. Its computational complexity is determined by two main components: the search for the first point of the object and the sequential search for objects. The advantage of the algorithm with respect to the problem under consideration is that it isolates only the outer boundary of the object, without separating the internal ones.

Construction of convex shells of perlite grains. There are many algorithms for extracting a convex hull, for example, the algorithm of Chan, Kirkpatrick, Melkman [21], but Graham [22], Jarvis [23] and the so-called "quickhull" (QH) algorithms were most widely used [24]. Their effectiveness has been investigated for the problem on binary images of simple figures and pearlite spots [25]. On simple figures, all algorithms showed an adequate result with a slight difference in speed. On binary images of real objects - pearl spots obtained from images of microstructures of metal pipelines, the Jarvis algorithm and the QH algorithm distinguish the convex envelopes of the spot correctly, unlike the Graham error algorithm. In this case, the average time of the Graham algorithm was approximately 1.1 times less than the QH algorithm and 1.9 times less than the Jarvis algorithm (the experiment was performed on PC with AMD Athlon II X2 3GHz and 3GB RAM). Therefore, the method included the QH algorithm. Note that the computational complexity of the Graham algorithm does not depend on the number of vertices found and is proportional to $q \log (q)$, where $q$ is the number of external points of the polygon (spot). The complexity of the Jarvis algorithm depends on the number of vertex spots and is proportional to qh, where $h$ is the number of common spot points and its convex hull, which in the worst case is $q^{2}$. The computational costs of the QH algorithm is compounded by the complexity of constructing all subsets. At best, the problem is divided into two equally powerful subtasks, then the complexity of the algorithm is from $2 q$ to $q^{2}$. The advantage of the $\mathrm{QH}$ algorithm is also the possibility of parallel computations for all subsets.

Examples of isolated convex shells of pearlite spots are shown in Fig. 4, a. After the convex hulls are selected, their linear characteristics are calculated, which are then used to estimate the microstructural parameters.

\subsection{Estimation of microstructural parameters}

In order to find the microstructural parameters of the spots, stochastic gradient descent-based estimation was used [14]. The point is that the parameters of templates are adaptive and adapt to the parameters of the spots represented by convex hulls. The initial approximations of the parameters of the templates are chosen taking into account the working range of the stochastic gradient descent optimization procedures. As an a priori information for finding the initial approximations of the parameters of the templates, the area of each selected convex hull in the image is used, which is related to the area of the desired ellipse by the obvious relation: $S=\pi \mathrm{ab}$. Studies have shown that three initial approximations of ellipticity (semi-axis relations) are sufficient: $\mathrm{c}=(\sqrt{3})^{-1}, 1$ and $\sqrt{3}$. In this case, taking into account that the ellipse at $\mathrm{c}=(\sqrt{3})^{-1}$ differs from the ellipse $\mathrm{c}=\sqrt{3}$ only by a rotation by $90^{\circ}$, we obtain as the initial approximations the circle $\left(c_{0}=1, \phi_{0}=0^{0}\right)$ and two ellipses $\left(c_{0}=1 / 3, \phi_{0}=0^{0}\right)$ and $\left(c_{0}=1 / 3\right.$, $\left.\phi_{0}=90^{0}\right)$.

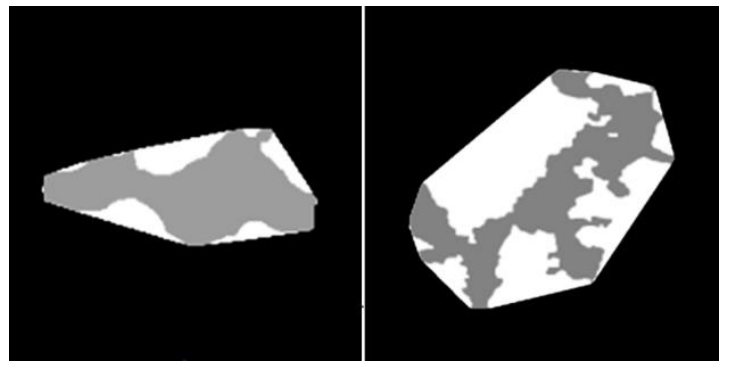

a)

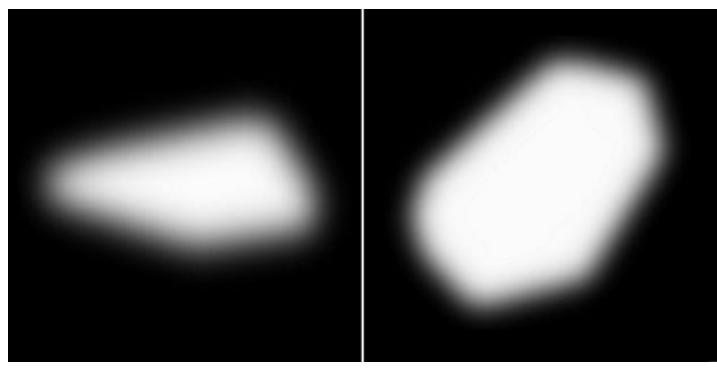

b)

Fig. 4. Examples of convex shells of pearlitic spots and the results of their filtering.

In order to increase the working range of the evaluation, the templates and convex hulls obtained for each object under study are subjected to an approximate procedure [18] of Gaussian filtering with a filter radius of $15 \%$ of the linear dimension of the object. Examples of filtered convex shells of pearlite spots are shown in Fig. 4, b. 
As a model of possible deformations of a customized template, when it is adjusted to a convex hull, a model similar to the similarity model is used:

$$
\dddot{x}=x_{0}+\kappa\left(\left(x-x_{0}\right) \cos \phi-k\left(y-y_{0}\right) \sin \phi\right)+h_{x}, y=y_{0}+\kappa\left(\left(x-x_{0}\right) \sin \phi+k\left(y-y_{0}\right) \cos \phi\right)+h,
$$

where as the following adaptive parameters are used: scale factor $\kappa$, ellipticity coefficient $k$, parallel shift $\bar{h}=\left(h_{x}, h_{y}\right)$, $\phi$ - directional angle and the coordinates of the rotation center of the spot $\left(x_{0}, y_{0}\right)$ [26]. It should be noted that to ensure the work of stochastic gradient descent optimization procedures, it is necessary to estimate all the coefficients of the model, and to calculate the microstructural parameters it is sufficient to use just $k$ and $\phi$. For each $i$-th spot with respect to the parameters of the adapted template, which has the maximum correlation with its convex hull, the following parameters are calculated: longitudinal size $W_{i}$; transverse size $H_{i}$; average size $d_{i}$; directional vector $\vec{K}_{i}^{\text {dir }}$ and the form anisotropy coefficient $k$ (which coincides in this technique with the ellipticity coefficient). For example, Fig. 5 shows the histograms of the distribution of spots by the coefficient of anisotropy $k$ of the shape (fig. 5,a) and the directional angle (fig. 5,b). The left figure corresponds to the left microstructure of fig. 3,b, and the right one - the right. The selected type of histogram of grain directivity (from 0 to 180 degrees), due to the specific nature of the problem, makes it possible to identify the directions of growth of pearlite spots close to 0 (180) degrees.

Then the integral parameters of the grains are found: the number of grains, the ratio of perlite to ferrite, the granularity of the pearlite phases, the general grain orientation vector, the average grain size, the grain size distribution, the degree of ordering of the grain orientations, and the mean value of the anisotropic shape coefficient. In particular, for the left image of fig. 3,b, we obtain: $n=41, k_{\mathrm{PF}}=31,3 \%, k_{\text {grit }}=26,5 \%, \bar{d}=43, \delta_{d}=37, \bar{k}_{\text {end }}=0,44,\left|\vec{K}_{\Sigma}^{\text {dir }}\right|=696, \phi=168, k_{\text {ord }}=0,39$, and for the right one: $n=30$, $k_{\mathrm{PF}}=32,7 \%, k_{\text {grit }}=28,5 \%, \bar{d}=34,2, \delta_{d}=23, \bar{k}_{\text {end }}=0.40,\left|\vec{K}_{\Sigma}^{\text {dir }}\right|=718, \phi=70, k_{\text {ord }}=0,70$. From the obtained strength characteristics, taking into account the data from [12], it can be concluded that the metal structure shown in fig. 3,b on the left is equivalent to cold rolling with a coefficient of deformation $\varepsilon=0,3$, and in fig. 3,b on the right $\varepsilon=0,47$ with $\varepsilon_{\text {crit }}=0,5$. Due to minor deviations from the factory parameters, the product with the microstructure shown in the left image in fig. $3 \mathrm{~b}$ can be allowed for further operation, but with the microstructure shown in the right-hand image of fig. $3 \mathrm{~b}$, taking into account the grain parameters, orientation and anisotropy of the grain shape, requires an additional, more in-depth analysis of the metal.
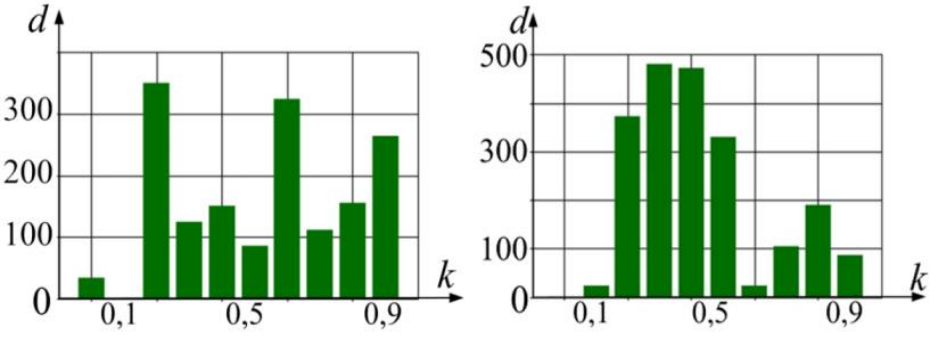

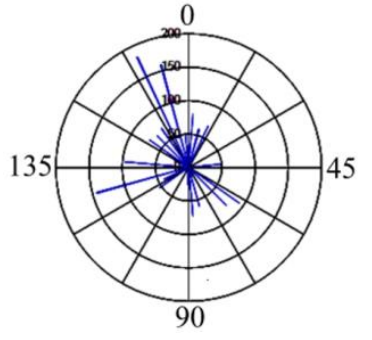

b)

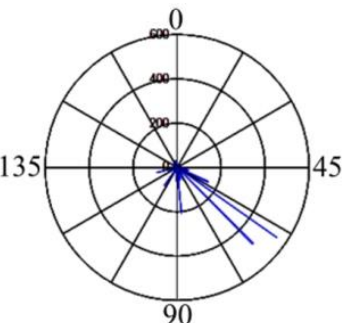

90

Fig. 5. Histograms of the shape anisotropy coefficient and the grain directivity angle.

It should be noted that the conducted studies showed that the average grain sizes, automatically found using the method examined, and calculated according to the traditional methods of GOST 5639 [6] differ by no more than 5\%.

As already noted, the practical actual task is to determine the changes in the strength characteristics of metals after a long period of operation. A particularly topical task is to determine the changes in the strength characteristics of metals, after prolonged use. The processes of changing the microstructure of metal structures during long-term operation are similar to the processes of cold rolling metals with a certain degree of deformation. We will give an example of the change in the microstructural characteristics of the steel of the 12GS pipeline after 40 years of explantation with its outer (fig. 6,a) and internal (fig. 6,b) surfaces. Histograms of the directivity vectors of microstructures are also shown there.
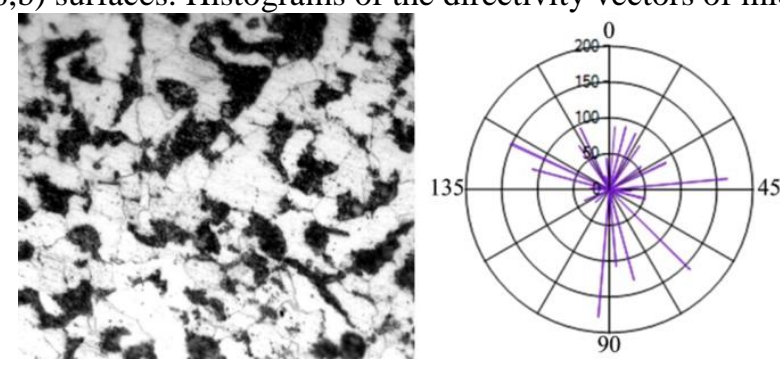

a)
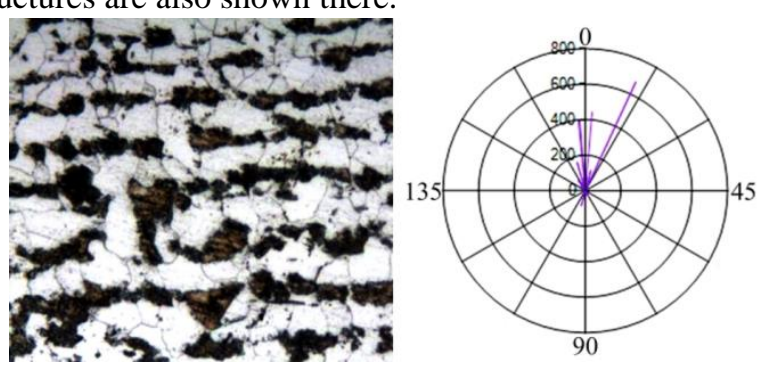

b)

Fig. 6. The microstructure of $12 \mathrm{GS}$ steel after 40 years of operation and its histogram of directional vectors external (a) and internal (b) pipeline surfaces.

Analysis of the data shows that all the parameters of the microstructure, other than the directivity, as well as the strength characteristics of the outer and inner surfaces of the pipeline metal differ slightly. It is essential to differentiate the orderliness of the microstructure grains, which on the one hand leads to hardening, but on the other hand to the brittleness of the metal and the 
increase in the probability of the appearance of microcracks. Therefore, the forecast of the strength characteristics of the pipeline metal must take into account the directivity of the grains of both the external and internal surfaces.

\section{Conclusion}

A technique for determining the microstructural parameters of low-carbon steel is proposed. It allows to determine from metallographic images in real time the perlite to ferrite ratio, perlite grain, the general grain orientation vector, the average grain size, the grain size distribution, the degree of ordering of the grain orientations, and the mean value of the anisotropic shape coefficient.

The technique can be arbitrarily divided into three stages: preliminary processing of the images under study, aimed at increasing the accuracy and reliability of finding microstructural parameters, determining the areas corresponding to perlite grains on the images, and actually evaluating the microstructural parameters of the grains. Preprocessing includes operations: color reduction, selection of the working area of processing, image filtering to compensate for high-frequency distortion caused by the peculiarities of the metallographic microscope path, compensation of uneven illumination of the microsection, histogram equalization. Segmentation of perlite grains on the images is achieved by the following procedures: segmentation, aimed at identifying areas of pearlite grains, mathematical morphology for eliminating internal discontinuities in grain images and excluding from the further analysis of small objects, delineating outer boundaries and constructing convex shells of grains. Estimation of microstructural parameters includes the formation of adaptive templates for finding object parameters, Gaussian filtering of convex shells of segmented grains and formed templates for the purpose of expanding the working range of the required parameters, finding the microstructural parameters of the perlite grains and the degree of granularity of the pearlite phases.

The peculiarity of the technique is that the parameters of the templates are adaptive and adapt to the parameters of the spots represented by convex hulls. As an a priori information for finding the initial approximations of the parameters of the templates, the area of the selected convex envelope of the spot is used.

The proposed technique can be used to determine the strength characteristics of the metal at different stages of production and operation: from quality control at the plant to determining the remaining resource. Approbation of the technique on images of microsections of oil and water pipelines of different service life has shown its high efficiency.

\section{Acknowledgement}

The reported study was supported by RFBR and Government of Ulyanovsk region (Russia), project № 16-41-732053.

\section{References}

[1] Garber EA. Cold rolling mills: (theory, equipment, technology). Cherepovets: CSU HPE, 2004; 416 p. (in Russian)

[2] Arzamasov BN, Makarova VI, Muhin GG, Rizhov MN, Silaeva VI. Material Science: A Textbook for Universities. 8 th ed. A stereotype. Moscow: Publishing House of the Moscow State Technical University, 2008; 648 p. (in Russian)

[3] GOST 380-2005 Carbon steel of ordinary quality. Stamps. Moscow: "Standardinform" Publisher, 2008; 5 p. (in Russian)

[4] GOST 5640-68 Steel. Metallographic method for evaluating the microstructure of sheets and ribbons. Moscow: Publishing house of Standards, $1988 ; 17$ p. (in Russian)

[5] Starodubov DN. Methods and algorithms for processing and analysis of flaw detection and metallographic images: cand. tech. sciences thesis: 05.13.01. Vladimir, 2008; 183 p. (in Russian)

[6] GOST 5639-82 Steels and alloys. Methods for the detection and determination of grain size. Moscow: IPK Publishing House of Standards, 2003; 20 p. (in Russian)

[7] Gumerov AG, Zainullin RS, Yamaleev KM, Roslyakov AV. The aging of the pipes of oil pipelines. Moscow: "Nedra" Publisher, 1995; 218 p. (in Russian)

[8] Malygin GA. Plasticity and Strength of Micro- and Nanocrystalline Materials (Review). Physics of the solid body 2007; 49(6): 961-982. (in Russian)

[9] Vinogradova LA, Magdeev RG, Kurganova YuV. Algorithm for the determination of the ratio of phase shapes in the perlite of tubular steels with the structure of ferrite and perlite. RVM 2012; 6: 41-45. (in Russian)

[10] Magdeev RG, Vinogradova LA, Dementiev VE. Method for determination of graininess in the pearlitic constituent of metals using imaging methods. Bulletin of Ulyanovsk State Technical University 2010; 4: 40-42. (in Russian)

[11] Tretyakov AV, Trofimov GK, Guryanova MK. Mechanical properties of steels and alloys during plastic deformation. Pocket Guide. Moscow: "Mechanical Engineering", 1971; 63 p. (in Russian)

[12] Vinogradov AI, Traino AI, Sarycheva IA. On the transformation of the grain structure of a metal during plastic deformation. Metals 2009; 2: 54-60. (in Russian)

[13] Sarycheva IA. Method and algorithms for processing information for evaluating the mechanical characteristics of cold-rolled carbon steels: cand. tech. sciences thesis: 05.13.01. Cherepovets, 2012; 130 p. (in Russian)

[14] Sadykov SS, Starodubov DN. Algorithms for determining the length and width of discrete area objects. Automation and modern technologies. Moscow: Mechanical Engineering, 2007; 10: 8-12. (in Russian)

[15] NEXSYS ImageExpert ${ }^{\mathrm{TM}}$ Pro 3: Program for quantitative analysis of images. URL: http://www.nexsys.ru/nexsys_iepro3x.htm (14.02.2017).

[16] GOST 8233-56 Steel. Standards of microstructure. Moscow: IPK Publishing House of Standards, 2004; 10 p. (in Russian)

[17] Tashlinsky AG. Estimation of the parameters of spatial deformations of image sequences. Ulyanovsk: UISTU, 2000; 132 p. (in Russian)

[18] Tashlinskii AG. Pseudogradient Estimation of Digital Images Interframe Geometrical Deformations. Vision Systems: Segmentation \& Pattern Recognition. Vienna, Austria: I Tech Education and Publishing 2007: 465-494. DOI: 10.5772/4975.

[19] Gonzalez RC, Woods RE. Digital Image Processing. 3rd Edition. New Jersey: Prentice-Hall Inc., 2006; 921 p.

[20] Wizil'ter YuV, Zheltov SYu, Bondarenko AV, Ososkov MV, Morzhin AV. Processing and analysis of images in computer vision problems: A course of lectures and practical exercises. Moscow: "Fizmatkniga" Publisher, 2010; 672 p. (in Russian) 
Image Processing, Geoinformation Technology and Information Security / R.G. Magdeev, A.G. Tashlinskiy

[21] Dementiev VE, Magdeev RG, Dementiev EG. Use of image processing algorithms for inspection of steel pipelines. Survey of buildings and structures: problems and solutions: materials VI int. Scientific-practical. Conf., October 15-16 - St. Petersburg: Publishing house Polytechnic. University, 2015; 5868. (in Russian)

[22] Gruzman IS, Kirichuk VS, Kosykh VP, Peretyagin GI, Spektor AA. Digital image processing in information systems: Proc. Allowance. Novosibirsk: Publishing house of NSTU, 2002; 352 p. (in Russian)

[23] Baatz M, Schäpe A. Multiresolution Segmentation: an optimization approach for high quality multi-scale image segmentation. Journal of Photogrammetry and Remote Sensing 2004; 58(3-4): 239-258.

[24] Magdeev RG, Tashlinsky AG. Efficiency of object identification on binary images using pseudo-gradient adaptation procedures. Radio engineering 2014; 7: 96-102. (in Russian)

[25] Kormen TH, Leiserson CE, Rivest RL, Stein C. Introduction to Algorithms. 3rd Edition. Boston: MIT Press, 2009; 1312 p.

[26] Williams DJ, Shas M. Edge Contours Using Multiple Scales. Computer Vision, Graphics and Image Processing 1990; 256-274. DOI: 10.1016/0734.

[27] Graham RL. An efficient algorithm for determining the convex hull of a finite planar set. Information Processing Letters 1972; 1: 132-133. DOI: $10.1016 / 0020$.

[28] Barber CB, Dobkin DP, Huhdanpaa H. The quickhull algorithm for convex hulls. ACM Transactions on Mathematical Software 1996; 22(4): 469-483. DOI: $10.1145 / 235815$.

[29] Magdeev RG, Biktimirov LSh. Application of algorithms for constructing a convex hull in the analysis of images of a metal microstructure. News of the Samara Scientific Center of the Russian Academy of Sciences 2014; 16(2): 496-500. (in Russian)

[30] Magdeev RG, Tashlinskii AG. A comparative analysis of the efficiency of the stochastic gradient approach to the identification of objects in binary images. Pattern recognition and image analysis 2014; 24(4): 535-541. DOI: 10.1134/S1054661814040130. 